\title{
Desarrollo de correlaciones para determinar el potencial a licuefacción mediante la aplicación del ensayo de penetración estática de piezocono (CPTu) en una cámara de calibración (CC) Development of correlations to determine the liquefaction potential by applying the piezocone test (CPTu) in a calibration chamber (CC)
}

Sebastián Rodríguez-Rodríguez ${ }^{1}$

Rodríguez-Rodríguez, S. Desarrollo de correlaciones para determinar el potencial a licuefacción mediante la aplicación del ensayo de penetración estática de piezocono (CPTu) en una cámara de calibración (CC). Tecnología en Marcha. Vol. 33, especial Movilidad estudiantil. Pág 91-103.

doi https://doi.org/10.18845/tm.v33i7.5482 


\title{
Palabras clave
}

Ensayo penetración estática de piezocono (CPTu); Resistencia de cono en punta qc; Densidad relativa ID; Ensayo triaxial cíclico no drenado; Relación de resistencia cíclica (CRR); Licuefacción.

\section{Resumen}

El objetivo principal de este proyecto fue desarrollar correlaciones para determinar el potencial a licuefacción de depósitos de arenas sueltas empleando el ensayo de penetración estática de piezocono CPTu; para ello fue necesario determinar el comportamiento de ensayos (CPTu) en la cámara de calibración (CC) del Laboratorio de Mecánica de Suelos y Rocas (IBF) en el Instituto Tecnológico de Karlruhe, además determinar la resistencia a licuefacción por medio de ensayos triaxiales cíclicos no drenados para muestras de arena, plantear una correlación entre la densidad relativa de almacenamiento (ID) de la muestra, la resistencia de punta (qc) del CPTu y la resistencia al flujo (CRR) de los ensayos triaxiales y por último comparar la correlación experimental obtenida con correlaciones existentes de la literatura. Con este proyecto se concluyó que en la región de bajas densidades de almacenamiento y bajas resistencias en punta una buena similitud, esto al comparar las correlaciones para los materiales experimentales estudiados por el IBF con correlaciones provenientes de la literatura. Ahora bien, para densidades altas y, por lo tanto, mayores resistencias en punta, las curvas de correlación para los materiales experimentales fueron más planas que en referencias bibliográficas; en otras palabras, la resistencia a licuefacción de los materiales experimentales en esta área es menor de lo que habrían predicho las curvas de la literatura. Así pues, no es posible aplicar directamente los resultados de este proyecto a otros sitios de estudio; ya que los materiales de las correlaciones realizadas (Kali4) y otros suelos difieren en aspectos como la mineralogía (criterios geológicos), la granulometría y la forma de partícula (criterios de composición); los cuales influyen sobre la susceptibilidad a la licuación.

\section{Keywords}

Cone penetration test with pore pressure measurement (CPTu); Cone tip resistance qc; Relative density ID; Cyclic undrained triaxial test; Cyclic resistance ratio (CRR); Liquefaction.

\begin{abstract}
The main objective of this project was to develop correlations to determine the liquefaction potential of loose sand deposits, using the CPTu test; for this purpose, it was necessary to determine the behavior of CPTu tests in the calibration chamber (CC) of the Institute of Soil Mechanics and Rock Mechanics (IBF) in Karlsruhe Institute of Technology, moreover to determine the resistance to liquefaction by means of cyclic undrained tests for sand samples, also to establish a correlation between the relative storage density (ID) of the sample, the tip resistance (qc) of the CPTu and the liquefaction resistance (CRR) of the triaxial tests and finally compare the experimental correlation obtained with existing correlations of the literature. With this project it was concluded that when comparing the correlations for the experimental materials studied by the IBF, it was determined that in the region of low storage densities and low peak resistances a good similarity. However, for higher densities and, therefore, greater peak resistances, the correlation curves for the experimental materials were flatter than in bibliographic references. In other words, the liquefaction resistance of the experimental materials in this area is lower than what the curves of the literature would have predicted. Finally, it is not possible to directly apply the results of this project in any other sand deposit; since the materials of the correlations made (Kali4) and other materials differ in aspects such as mineralogy (geological criteria), granulometry and particle form (composition criteria); which influence the susceptibility to liquefaction.
\end{abstract}




\section{Introducción}

Según Ishihara [1], el fenómeno de licuefacción se manifiesta cuando un depósito de arena saturada se ve sometido a ondas sísmicas de corte que se propagan durante un sismo; de manera que la estructura de la arena tiende a densificarse. Por consiguiente, la duración de la aplicación de las tensiones de corte cíclico es, en general muy pequeña en comparación con el tiempo necesario para que ocurra algún drenaje, por esta razón la tendencia de la arena a disminuir de volumen durante cada ciclo se refleja en un incremento progresivo de la presión de poros. Esto produce una reducción continua de la tensión efectiva y consecuentemente una disminución en la resistencia al corte de la arena. Si la arena se encuentra en estado suelto, la presión de poros puede incrementarse rápidamente a un valor igual a la presión de confinamiento, y la capa de suelo puede experimentar grandes deformaciones. En otras palabras, la arena sufre licuefacción, esto es, se comporta como un líquido viscoso, generándose grandes deformaciones que pueden dar origen a múltiples mecanismos de falla, algunas de ellas catastróficas como por ejemplo: falla de taludes, el rompimiento de presas y la destrucción de puentes (figura 1).

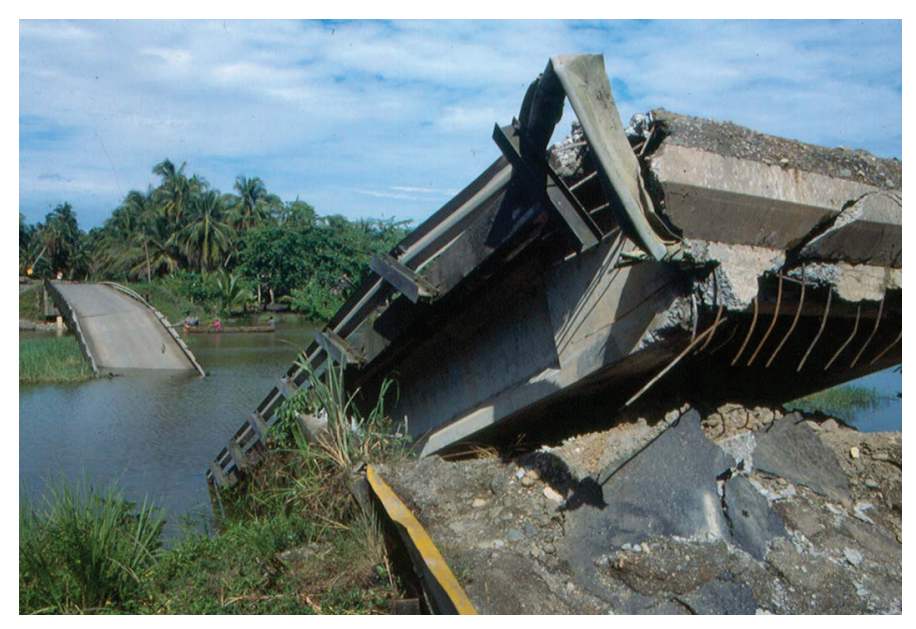

Figura 1. Efectos de licuación de suelos por el terremoto de Limón en 1991. Fuente: [2].

La resistencia a licuefacción del suelo puede estimarse a partir de ensayos triaxiales cíclicos no drenados, en la medida de lo posible en muestras no alteradas o bien realizando reconstrucción de muestras en laboratorio. Alternativamente, es posible utilizar diagramas que relacionan la resistencia a licuefacción en función de la velocidad de la onda de corte o bien la resistencia en punta en ensayos de penetración como se ve en las figuras 2 y 3 (resistencia en punta máxima del ensayo CPT o bien número de impactos NSPT en el ensayo SPT). Estos diagramas de correlación han sido construidos basándose en la recolección de datos en campo (factores de sondeo, velocidad de onda de corte). Por ende solamente son aplicables a suelos en estado natural; por lo tanto, no pueden ser simplemente transferidos a arenas sueltas. Así entonces para deducir la susceptibilidad al flujo de depositos de suelos sueltos se deben desarrollar diagramas que correlacionen la resistencia al flujo para material extraído. 


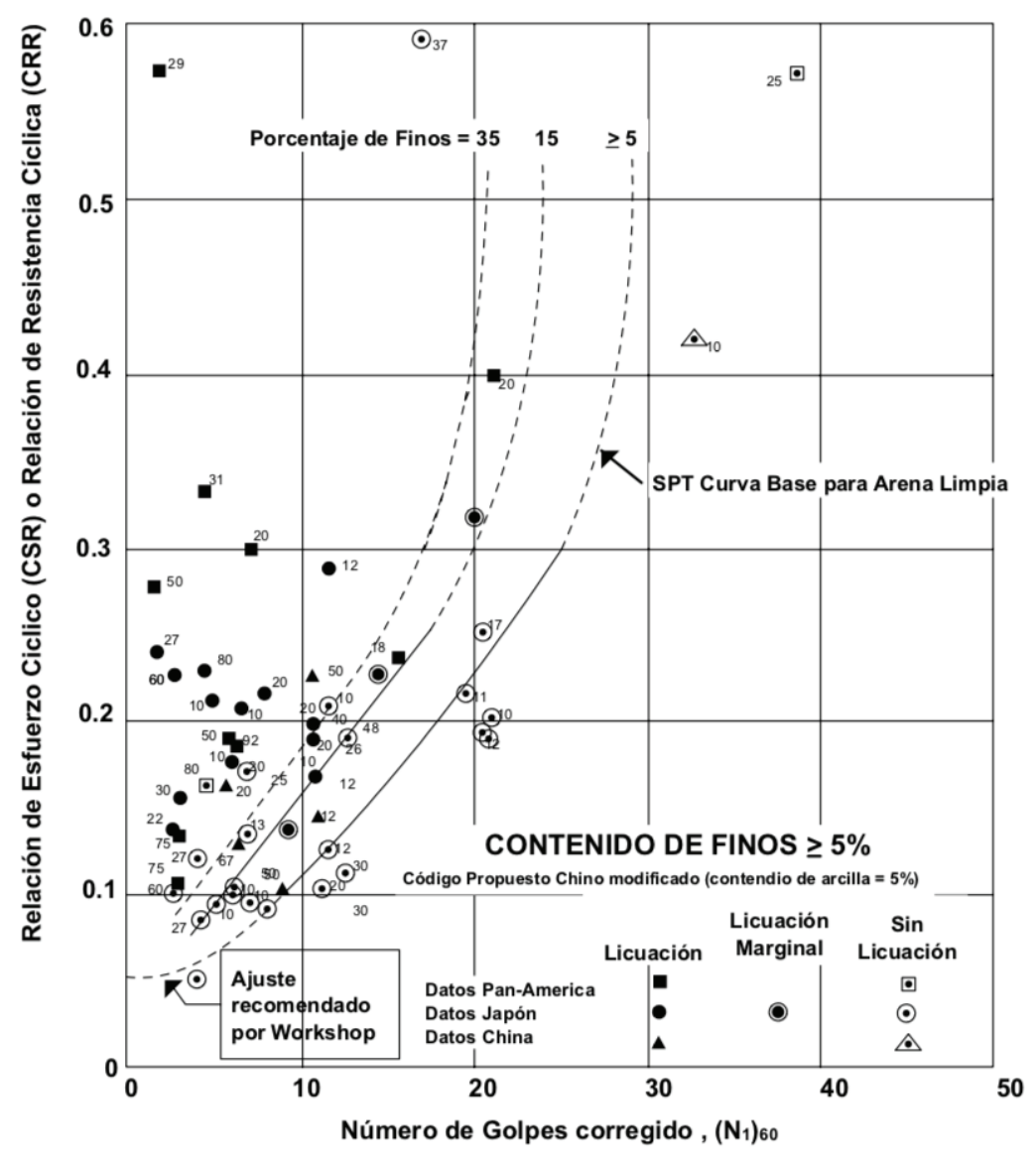

Figura 2. Relación entre la resistencia a licuefacción CRR en función del número de impactos SPT (N1)60. Fuente: [3].

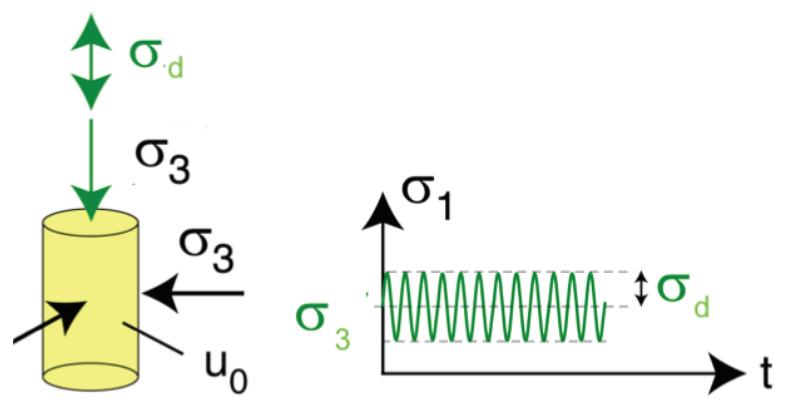

Figura 3. Relación entre la resistencia a licuefacción CRR en función de resistencia en punta qc. Fuente: [3].

Este artículo resume una serie de ensayos llevados a cabo en el Laboratorio de Mecánica de Suelos del Instituto de Mecánica de Suelos y Mecánica de Rocas del Instituto Tecnológico de Karlsruhe, también conocido como IBF por sus siglas en alemán (Institut für Bodenmechanik und Felsmechanik). Se propuso desarrollar correlaciones para determinar el potencial a licuefacción de depósitos de una muestra de arena variando su densidad y su contenido de humedad empleando el ensayo CPTu; con este propósito se planteó: determinar el comportamiento de ensayos de penetración estática de piezocono (CPTu) en la cámara de calibración (CC) del IBF, 
determinar la resistencia a licuefacción por medio de ensayos triaxiales cíclicos no drenados para muestras de arena, plantear una correlación entre la densidad relativa de almacenamiento (ID) de la muestra, la resistencia de punta (qc) del CPTu y la resistencia a licuefacción (CRR) (ver figura 3) de los ensayos triaxiales y por último comparar la correlación experimental obtenida con correlaciones elaboradas previamnete en el IBF y también con correlaciones existentes de la literatura.

\section{Materiales y métodos}

\section{Propiedades del material experimental}

Las pruebas en la cámara de calibración se han enumerado consecutivamente (Kali1, Kali2, Kali3 y Kali4). El material de prueba utilizado para este proyecto de graduación ha sido una arena tomada en junio 2017 de un campo de apilamiento de la explotación de carbón a cielo abierto; la cual se almacenó en varias bolsas grandes. Esta arena fue identificada como "Kali4".

Con el propósito de evitar algún efecto en los valores de resistencia en punta qc de materiales arcillosos o partículas de grava (con un diámetro mayor de 10,0 mm); se decidió eliminarlos antes del uso del material experimental Kali4 en la celda de calibración, además las arenas limpias presentan mayor susceptibilidad a ser licuadas y por lo tanto se podrían tener efectos sobre el estudio de licuefacción del material Kali4. Luego de la recolección de muestras en campo, se tomó una muestra de cada "Big Bag" después de una homogeneización para un análisis de granulométrico por tamizado. Las curvas resultantes de distribución de tamaño de grano de los cuatro materiales de prueba se muestran en la figura 4. Para todas las muestras de la campaña de recolección de especímenes (Kali's), se puede observar una clara dispersión de las curvas de distribución del tamaño de grano en la figura 4. Esto se puede explicar por el hecho de que el muestreo del material se ha realizado en diferentes sitios, fechas y profundidades.

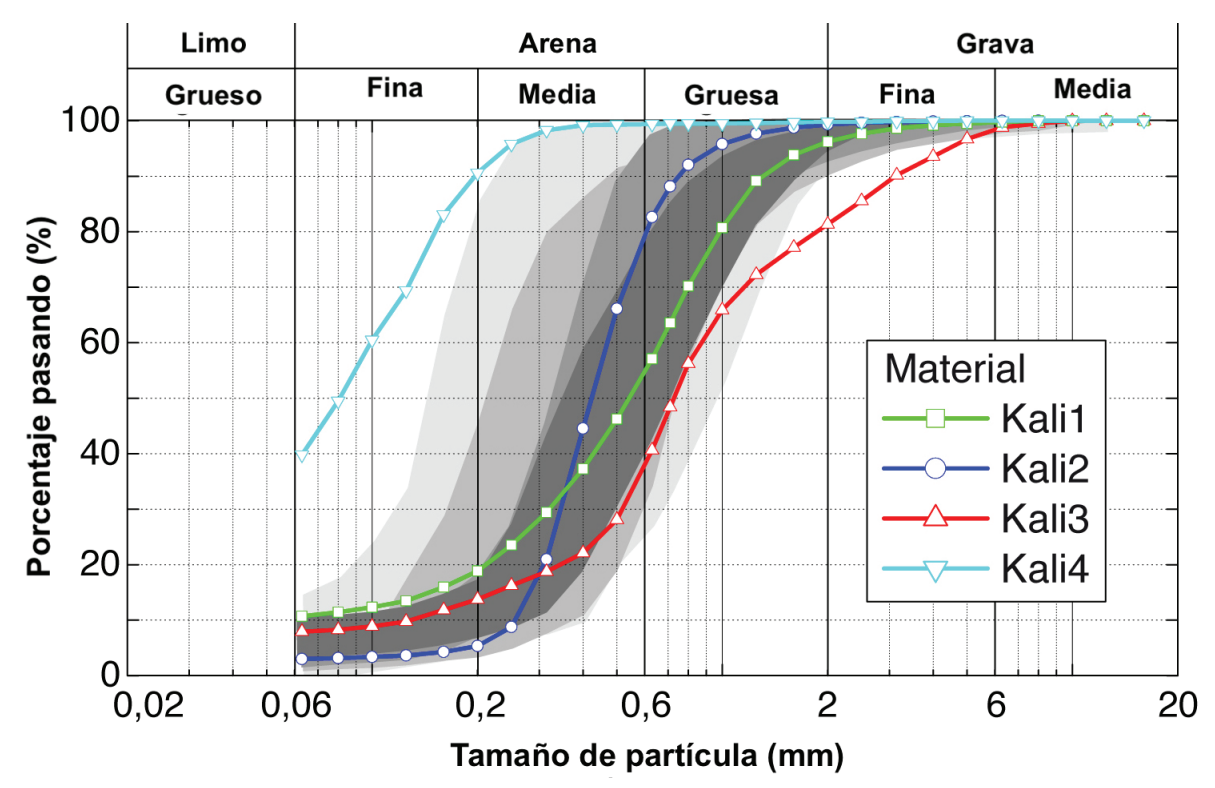

Figura 4. Curvas granulométricas de los materiales experimentales. Fuente: [4]. 
Cuadro 1. Propiedades físicas - mecánicas de los materiales de experimentales. Fuente: [5].

\begin{tabular}{|l|c|c|c|c|}
\hline \multirow{2}{*}{} & \multicolumn{4}{|c|}{ Arena } \\
\cline { 2 - 5 } & Kali1 & Kali2 & Kali3 & Kali4 \\
\hline Diámetro medio del grano d50 $(\mathrm{mm})$ & 0,54 & 0,42 & 0,73 & 0,116 \\
\hline Proporción de grano fino FC $(\%)$ & 10,7 & 3,0 & 7,9 & 39,7 \\
\hline Coeficiente de uniformidad Cu = d60 / d10 & 3,77 & 1,83 & 6,79 & - \\
\hline $\begin{array}{l}\text { Coeficiente de curvatura Cc }=\mathrm{d} 302 / \\
\left(\mathrm{d} 60^{*} \mathrm{~d} 10\right)\end{array}$ & 2,39 & 1,04 & 2,18 & - \\
\hline Densidad de grano ps $\left(\mathrm{g} / \mathrm{cm}^{3}\right)$ & 2,65 & 2,64 & 2,64 & 2,64 \\
\hline Densidad seca mínima $\mathrm{pd} \min \left(\mathrm{g} / \mathrm{cm}^{3}\right)$ & 1,472 & 1,431 & 1,607 & 1,431 \\
\hline Densidad seca máxima $\mathrm{pd} \max \left(\mathrm{g} / \mathrm{cm}^{3}\right)$ & 1,877 & 1,699 & 1,922 & 1,699 \\
\hline
\end{tabular}

Las arenas "Kali1", "Kali2", "Kali3" y "Kali4" difieren significativamente en la distribución del tamaño de grano. "Kali2" es un material grueso, "Kali1" es un medio de grano grueso, arena fina y arena gruesa, "Kali3" es una arena gruesa fina y de grano fino de tamaño medio y "Kali4" es una arena más fina que todas las arenas anteriores, la cual contiene aproximadamente un 40\% de finos (arcillas, limos y hasta posiblemente material orgánico). Según las especificaciones del Sistema Unificado de Clasificación de Suelos (SUCS), "Kali4" se considera una arena limoarcillosa, SM-SC o SU-ST según la norma DIN 18196. Los parámetros más importantes de los materiales experimentales se resumen en el cuadro 1.

\section{CPTu en la cámara de calibración}

Las cámaras de calibracioyn son equipos relativamente grandes (figura 5), y los ensayos en ellas son bastante costosos en tiempo y recursos. Por otra parte, las pruebas se realizan en muestras de arenas reconstituidas cuyas propiedades varían (densidad y humedad) respecto de los depósitos naturales de donde provienen [6]. Para este proyecto de graduación se llevaron a cabo un total de 14 ensayos con el material "Kali4". Como ya se había trabajado en numerosas ocasiones, el método de instalación en la CC fue el mismo para anteriores tesis de licenciatura e informes de labores del IBF. La preparación y ejecución de la prueba se puede resumir y dividir en los siguientes pasos:

- Mezclado de los materiales con hasta un contenido de humedad de aproximadamente $12 \%$ a $15 \%$.

- Instalación del material en la cámara de calibración (variando la densidad relativa de almacenamiento en la instalación desde 0,2 - 0,8).

- Ejecución de prueba (variación de la presión lateral).

- Eliminación y muestreo con determinación del contenido de humedad y la densidad de almacenamiento.

Un esquema del ensayo en la cámara de calibración se muestra en la figura 5. Las muestras de arena miden aproximadamente 1,0 $\mathrm{m}$ de diámetro y aproximadamente 1,5 $\mathrm{m}$ de altura dentro de la CC. Después de la colocación de la muestra se coloca una tapa que contiene una abertura para el paso de la sonda de presión. Las paredes laterales, el fondo de la CC y la tapa están revestidos con membranas de goma, a través de las cuales se pueden aplicar presiones a la muestra en las direcciones horizontal $(\sigma 2=\sigma 3)$ y vertical $(\sigma 1)$. De esta manera, se puede probar un estado de tensión isotrópico $(\sigma 1=\sigma 2=\sigma 3)$ así como anisotrópico $(\sigma 1 \neq \sigma 2, \sigma 3)$. Después de la aplicación de la presión, la prueba se lleva a cabo. 


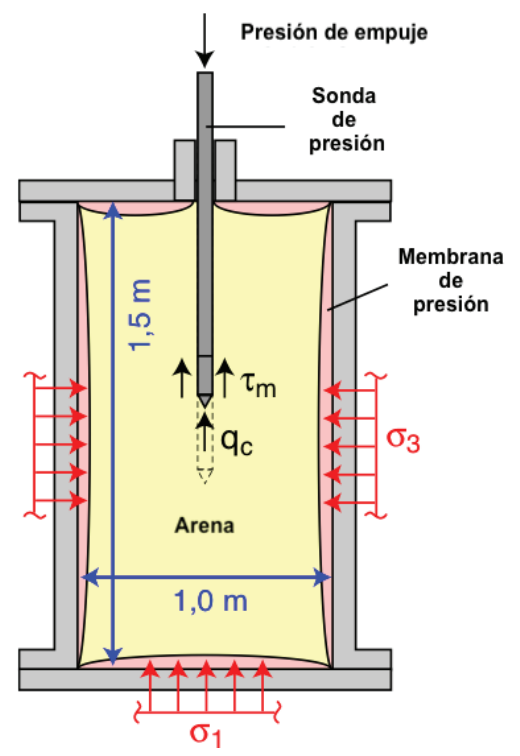

Figura 5. Esquema de la cámara de calibración del IBF. Fuente: [7].

En este proceso, una sonda de presión estándar (Van den Berg, Heerenveen) se introduce a través de la abertura en la tapa y posteriormente se introduce verticalmente en la muestra de suelo a una velocidad constante. La fuerza de inyección se genera por medio de un equipo hidráulico. Se mide la presión en punta qc, la fricción del manguito $\tau \mathrm{m}$ y la distancia vertical.

\section{Ensayo triaxial cíclico no drenado}

Con el fin de poder establecer correlaciones entre la resistencia en punta del CPTu y la resistencia a licuefacción, fue necesario aplicar carga cíclica no drenada en la arena experimental Kali4 con variación de la densidad de almacenamiento. Los ensayos han sido realizados por el personal del IBF.

Según [8] en un ensayo triaxial, un espécimen cilíndrico de suelo es revestido con una membrana de látex o caucho dentro de una cámara a presión. La parte superior e inferior de espécimen tiene discos porosos, los cuales se conectan al sistema de drenaje para saturar o drenar el espécimen. En el ensayo triaxial se puede variar la presión lateral actuante sobre la muestra de suelo en tres direcciones ortogonales, efectuándose así las mediciones sobre sus características mecánicas. El agua de la cámara puede adquirir cualquier presión deseada por la acción de un compresor. La carga axial se transmite al espécimen por medio de una barra que atraviesa la parte superior de la cámara. La presión que se ejerce con el agua que llena la cámara es hidrostática y produce, por lo tanto, esfuerzos principales sobre el espécimen, iguales en todas las direcciones. En una prueba triaxial de compresión, la presión axial siempre es el esfuerzo principal mayor, $\sigma 1$; los esfuerzos intermedios y menor son iguales $(\sigma 2=\sigma 3)$ y son iguales a la presión lateral. En un ensayo triaxial cíclico no drenado, se aplica un esfuerzo vertical total $\sigma 1$, una presión lateral total $\sigma 3$ y una presión de agua intersticial u (figura 6). El esfuerzo de tensión efectivo se convierte en $\sigma 1^{\prime}=\sigma-u$ y el esfuerzo lateral efectivo a $\sigma 3^{\prime}=\sigma 3$ - $u$. Durante el ensayo, la tensión lateral $\sigma 3$ se mantiene constante y la tensión vertical $\sigma 1$ con una amplitud oampl alrededor del valor medio olav es cíclicamente variada. Al comienzo del ensayo, la tensión inicial $\sigma 1=\sigma 1$ av corresponde generalmente a el esfuerzo lateral $\sigma 3$ (figura $6)$, es decir la condición de carga inicial es isotrópica. 


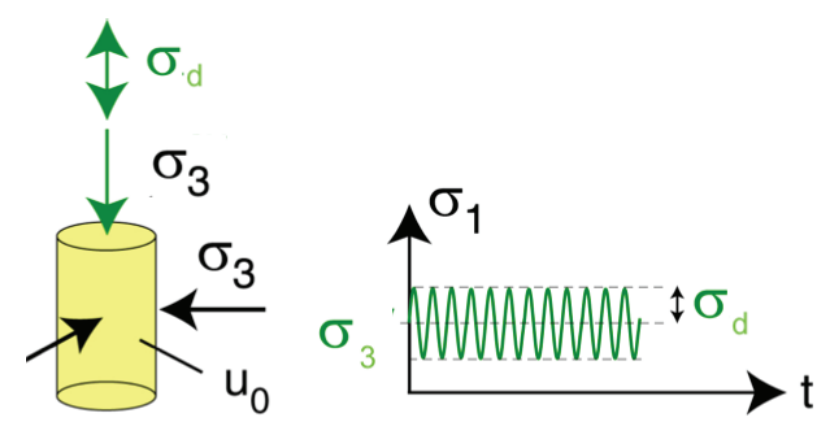

Figura 6. Estado de esfuerzos en el ensayo triaxial cíclico. Fuente: [7]

\section{Relación de Esfuerzos Cíclicos (CSR) y Relación de Resistencia Cíclica (CRR)}

Para evaluar la resistencia a licuefacción del suelo es necesario considerar dos variables: La demanda sísmica que se espera en las capas de suelo durante un sismo de diseño, expresada en términos de resistencia CSR (cyclic stress ratio). La resistencia de suelo para oponerse a la licuefacción, expresado con CRR (cyclic resistance ratio). El factor de seguridad frente a licuefacción puede definirse como el cociente CRR/CSR. Cuando el factor de seguridad contra licuefacción es menor que la unidad se presenta el fenómeno de licuefacción a la profundidad analizada (figura 7).

$$
\mathrm{FS}_{\text {lic }}=\frac{\mathrm{CRR}}{\mathrm{CSR}} \quad(\text { Ecuación 1) }
$$

Donde:

CRR: Relación de resistencia cíclica, representa la capacidad de resistencia del suelo. CSR: Relación de esfuerzo cíclico, representa la fuerza que actúa en el suelo.

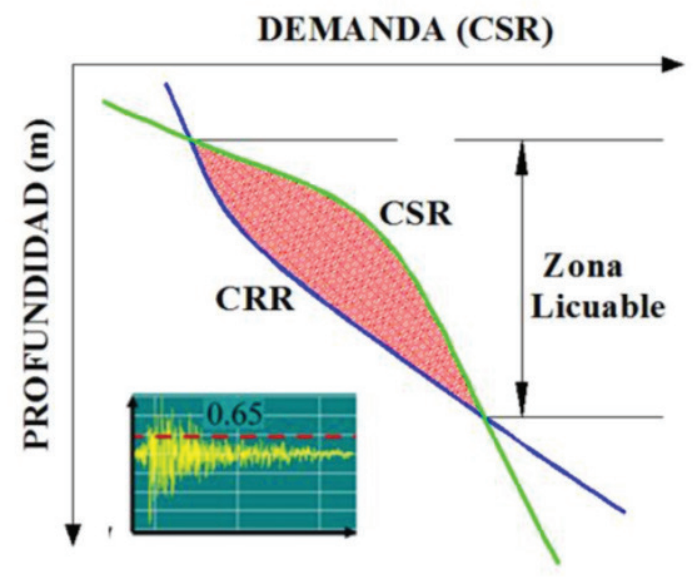

Figura 7. Esquema de relación de esfuerzo cíclico. Fuente: [9]. 


\section{Resultados}

Resultados de ensayos CPTu en la cámara de calibración del IBF para el material experimental Kali4

Con los datos para una presión de 100 kPa se desarrolló la figura 8; en donde se relaciona la densidad relativa de almacenamiento (ID) en función de la resistencia en punta del ensayo CPTu (qc1); esta función se registró por medio de la ecuación 2. La cual será utilizada en los siguientes apartados para generar la correlación entre qc1-ID y la resistencia a licuefacción CRR-ID.

$$
\mathrm{I}_{\mathrm{D}}=0,0147+0,1674 \mathrm{q}_{-\mathrm{c} 1}-0,0091 \mathrm{q}_{\mathrm{c} 1}^{2}, \mathrm{R} 2=0,8527 \quad \text { (Ecuación 2) }
$$

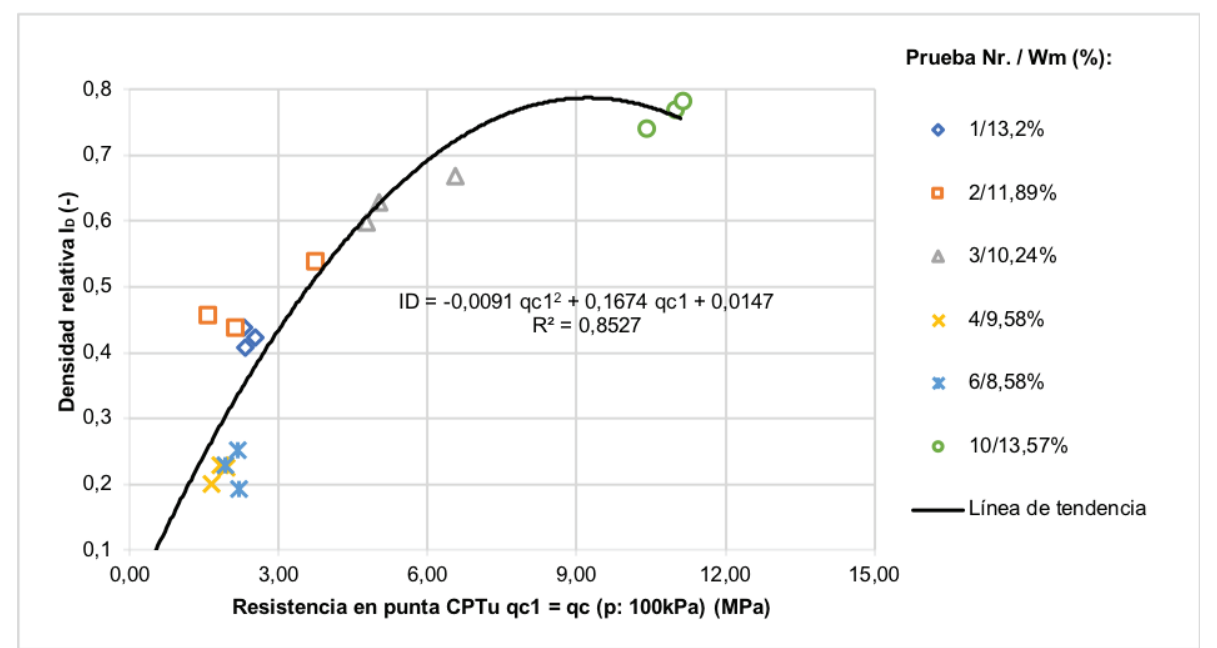

Figura 8. Densidad relativa de almacenamiento en función resistencia en punta qc en los ensayos en la CC para el material "Kali4". Fuente: Diagrama elaborado en Microsoft Excel por el autor.

Resultados de ensayos triaxiales cíclicos no drenados para el material experimental Kali4 La relación de resistencia cíclica $(\mathrm{CRR}, \mathrm{Nf}=10)$ se representa gráficamente en la Figura 9 como una función de la densidad relativa de almacenamiento ID. Para la presión inicial experimentada, existe una relación lineal. Esta curva para la presión p'0 $=100 \mathrm{kPa}$ es necesaria en el siguiente apartado para el desarrollo del diagrama de correlación. La relación puede describirse mediante la siguiente ecuación:

$$
\operatorname{CRR}\left(\mathrm{N}_{\mathrm{f}}=10\right)=0,086+0,0531 \mathrm{I}_{\mathrm{D}}, \mathrm{R} 2=1,0 \quad \text { (Ecuación 3) }
$$




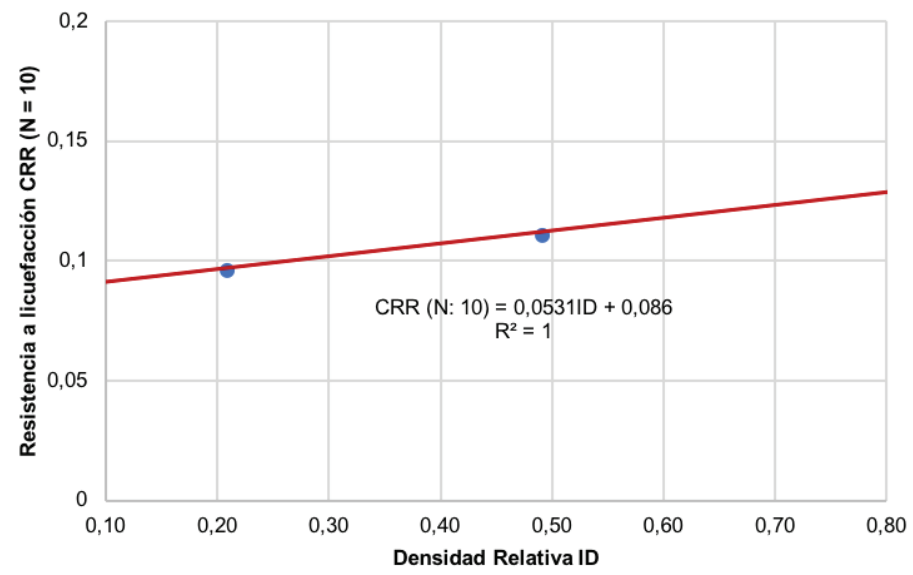

Figura 9. Relación de esfuerzos cíclicos CSR para licuefacción en 10 ciclos (CRR de resistencia a licuefacción) en función de la densidad relativa de almacenamiento inicial ID el material experimental "Kali4". Fuente: Diagrama elaborado en Microsoft Excel por el autor.

\section{Correlación entre la resistencia en punta normalizada qc1 y la relación de resistencia cíclica CRR para el material experimental Kali4}

Los resultados de los ensayos CPTu en la cámara de calibración y las pruebas triaxiales cíclicas no drenadas, han sido utilizados para establecer un diagrama de correlación entre la resistencia a licuefacción y la resistencia máxima en punta del CPTu. El diagrama se muestra en la figura 10. dado que los diagramas de correlación en la literatura siempre se configuran para una presión de referencia efectiva de $100 \mathrm{kPa}$, los datos experimentales para $p$ y p'0 $=100 \mathrm{kPa}$ también se emplearon en este caso.
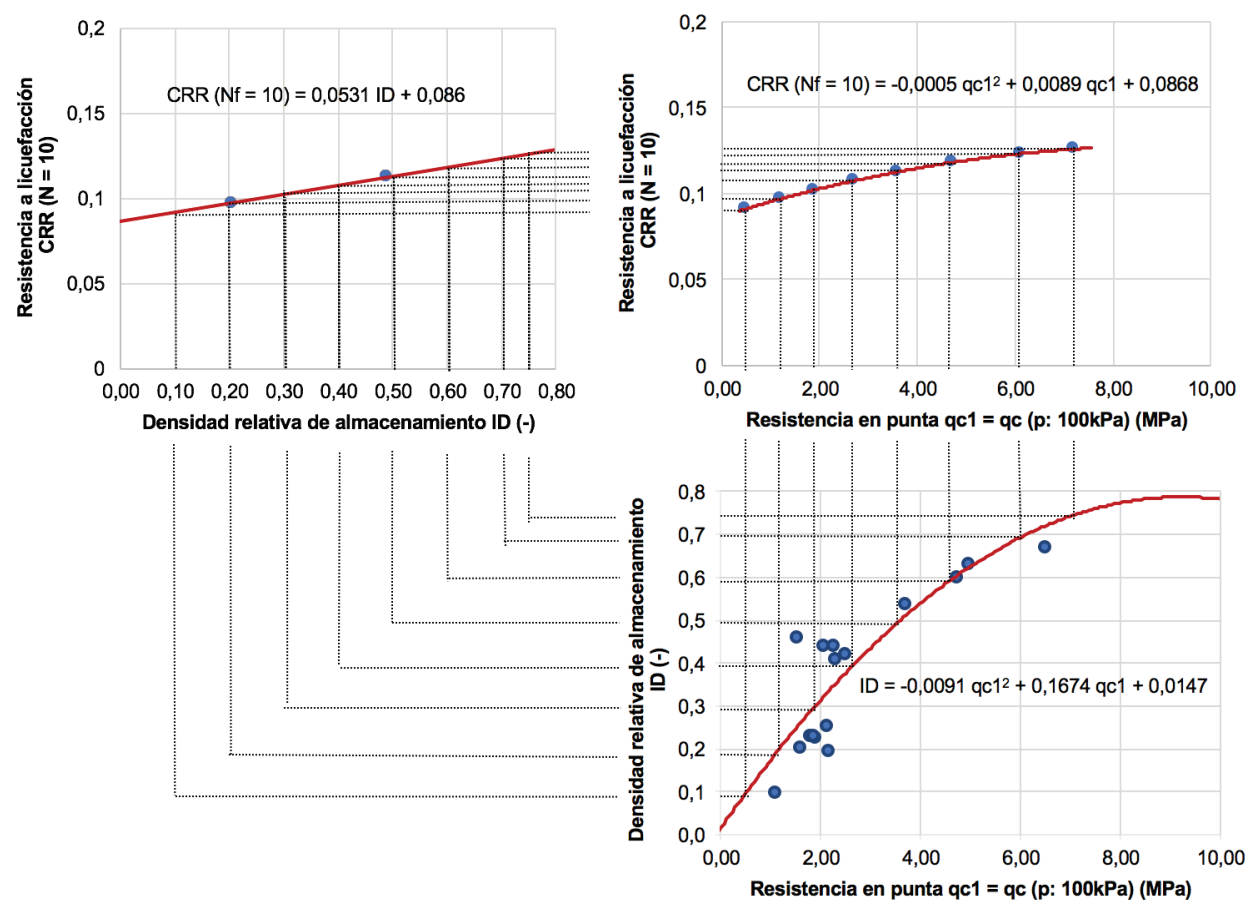

Figura 10. Correlación entre la resistencia a la licuefacción CRR para falla después de $\mathrm{Nf}=10$ ciclos y la resistencia en punta máxima normalizada $q c 1=q c\left(p^{\prime}=100 \mathrm{kPa}\right)$ para el material experimental "Kali4". Fuente: Diagrama elaborado en Microsoft Excel por el autor. 
La sección superior izquierda de la figura 10 proviene de los resultados de las pruebas triaxiales cíclicas no drenadas. Esta es la relación entre la resistencia a licuefacción por falla en 10 ciclos $(\mathrm{Mw}=7,5)$ y la densidad relativa de almacenamiento ID que se expresó en la figura 9. En la sección inferior derecha de la figura 10, se repite el resultado de los ensayos CPTu en la CC del IBF, mostrados en la figura 8.

En el diagrama de la esquina superior derecha de la figura 10, los valores se trazaron uno encima del otro, combinando así los pares (CRR, qc1) para las diferentes densidades de almacenamiento ID; donde se obtuvo una curva que describe la correlación entre la resistencia a licuefacción y la resistencia en punta máxima del ensayo CPTu para el material experimental "Kali4"; en fin, la relación entre la resistencia a licuefacción y la resistencia en punta del ensayo CPTu viene dado por:

$$
\operatorname{CRR}\left(\mathrm{N}_{\mathrm{f}}=10\right)=0,0868+0,0089 \mathrm{qc1}-0,0005 \mathrm{qc1} 1^{2}, \mathrm{R} 2=1,0 \quad \text { (Ecuación 4) }
$$

Comparación entre la correlación del material experimental y las existentes en la literatura

La figura 11 compara la correlación CRR - qc1 (figura 10) con las correlaciones de trabajos previos en la CC del IBF; además, se compara la correlación experimental obtenida con las correlaciones para arenas de origen natural provenientes de la literatura. Se distingue que la correlación del material experimental es significativamente más plana que las de la literatura. En el rango aproximado de resistencias en punta de $5 \mathrm{MPa} \leq \mathrm{qc} 1 \leq 7,5 \mathrm{MPa}$, se obtendrán resistencias a licuefacción similares entre la nueva correlación del material experimental Kali4 y las existentes. Para resistencias en punta más pequeñas que $5 \mathrm{MPa}$, la nueva correlación para el material experimental Kali4 proporciona resistencias a licuefacción más altas que las presentes en la literatura, así mismo resistencias a licuefacción más pequeñas a partir de un qc1 > 7,5 MPa.

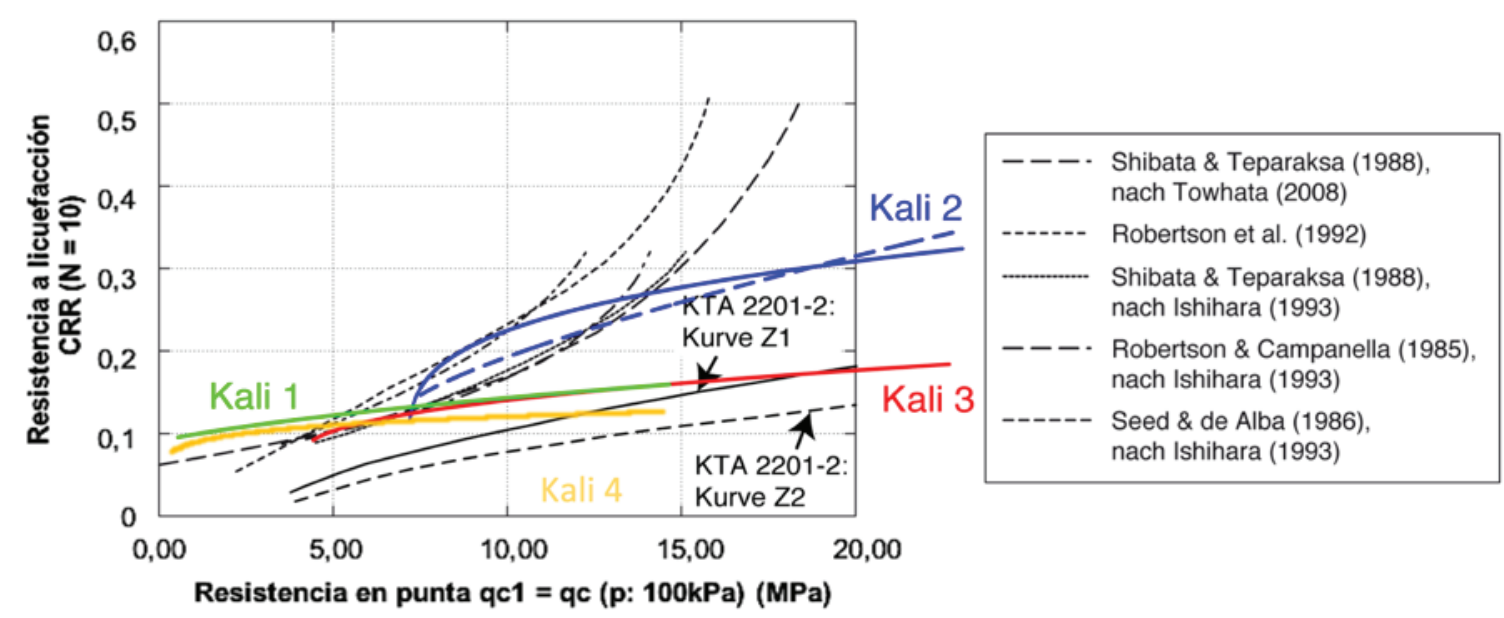

Figura 11. Comparación de la correlación CRR - qc1 para el material experimental Kali4 y trabajos previos con correlaciones presentes en la literatura para depósitos de arenas de origen natural. 


\section{Conclusiones}

- Se logró determinar que existe un aumento en la resistencia en punta qc con el aumento de la densidad relativa de almacenamiento ID y el aumento de la presión en la CC. Ambas dependencias fueron descritas por medio de ecuaciones.

- De acuerdo con los ensayos triaxiales cíclicos no drenados, la resistencia a licuefacción está influenciada principalmente por la densidad de almacenamiento ID; por lo tanto, a medida que aumenta la densidad relativa de almacenamiento ID, también aumenta la resistencia a licuefacción CRR $(\mathrm{Nf}=10)$.

- Según el diagrama de correlación CRR - qc1 para el material experimental Kali4, la resistencia a licuefacción aumenta al aumentar la resistencia en punta CPTu.

- Con este informe de proyecto de graduación se delimitó que la correlación elaborada depende significativamente del material experimental, donde la resistencia en punta del ensayo CPTu disminuye con el aumento del contenido de finos siendo consecuente con la literatura (Youd, y otros, 2001); por lo tanto, al material experimental "Kali4" se le relaciona con menores resistencias en punta. [10]

- Los resultados indican que también un alto nivel de grano fino da como resultado una baja resistencia a la licuefacción; determinando así que el material Kali4 tiene resistencias a la penetración de cono y a licuefacción menores que otros materiales experimentales estudiados anteriormente, esto por sus propiedades granulométricas lo cual concuerda con otras investigaciones realizadas en el IBF [11].

- Según la comparación de las correlaciones para los cuatro materiales experimentales estudiados por el IBF con correlaciones para suelos de origen natural, provenientes de la literatura, se determina que en la región de bajas densidades de almacenamiento y bajas resistencias en punta una buena similitud.

- Ahora bien, para densidades de almacenamiento densas y, por lo tanto, mayores resistencias en punta, las curvas de correlación para los materiales experimentales son más planas que las referenciadas, provenientes de la literatura. En otras palabras, la resistencia a licuefacción de los materiales experimentales en esta área es menor de lo que habrían predicho las curvas de la literatura ( [12], [13], [1], [14].

- La correlación realizada para el material Kali4 no es posible utilizarla para otros depósitos de suelos sueltos que no sean los materiales experimentales para la cual ha sido creada.

\section{Recomendaciones}

- Es importante el control de humedad de las muestras, por lo que se recomienda el uso de otro tipo de almacenamiento de la muestra experimental, ya que la perdida de humedad en los ensayos ha sido significativa.

- Sería satisfactorio para la metodología del ensayo CPTu en cámaras de calibración, realizar una normativa referente al ensayo CPTu para poder realizar verificaciones con otras investigaciones.

- Sería recomendable realizar mediciones de velocidad de onda cortante VS en la cámara de calibración y así crear correlaciones con estos resultados para la obtención de la densidad del material instalados en la CC.

- Se recomienda realizar algunas pruebas en la CC con un grado de saturación mayor, para analizar la presión de poros con el CPTu. 
- Se recomienda que se realicen los ensayos 8, 14 y 13 de la prueba CPTu ya que los resultados no concuerdan con la tendencia general y así poder corregir los errores provenientes de estas pruebas.

- Se recomienda el estudio detallado del estado de anisotropía en la CC, ya que aún no se ha realizado variedad de pruebas, además verificar algunas de las pruebas realizadas como por ejemplo el ensayo CPTu 14.

- Para futuros proyectos, también se podría dar uso de los resultados provenientes de la relación de fricción en el CPTu en la CC.

- Cuando se determine que un depósito de arena es susceptible a licuefacción, es necesario aplicar técnicas de drenaje y densificación de suelos, por ejemplo: explosiones controladas, vibro compactación.

- Es necesario y conveniente para el proyecto que se elaboren más ensayos triaxiales cíclicos no drenados con diversidad de densidades relativas, ya que para este informe de práctica profesional solamente se contó con 8 ensayos triaxiales para el material Kali4.

- No es posible aplicar directamente los resultados de este proyecto en Costa Rica; primeramente, porque en el país no se realizan proyectos de minería a cielo abierto; además los materiales de las correlaciones realizadas (Kali4) y los suelos en Costa Rica difieren en aspectos como la mineralogía (criterios geológicos), la granulometría y la forma de partícula (criterios de composición); los cuales influyen sobre la susceptibilidad a la licuefacción.

- Recomiendo adquirir este tipo de equipo para el desarrollo de investigaciones de depósitos sueltos de materiales en las zonas más susceptibles a licuefacción en Costa Rica.

\section{Referencias}

[1] K. Ishihara, «Soil Behaviour in Earthquake Geotechnics,» 1996.

[2] S. M. Castro, «Licuefacción de los suelos, típica de zonas con depósitos arenosos,» 1991.

[3] D. R. y A. T., «3rd Ishihara Lecture: An investigation into why liquefaction charts work: A necessary step toward integrating the states of art and practice,» 2015.

[4] W. T. y K. I. , Interviewees, Avances en resultados de la práctica profesional en el IBF. [Entrevista]. 10 Noviembre 2017.

[5] I. Kimmig, Interviewee, Realización de correlaciones entre CPTu y CRR. [Entrevista]. 2 Noviembre 2017.

[6] J. Butlanska, «Cone Penetration Test in a Virtual Calibration Chamber, Barcelona, 2014.

[7] W. T. y T. T., «Aufstellung einer Korrelation zwischen dem Spitzendruck aus Drucksondierungen und dem Verflussigungswiderstand eines Tagebau-Kippenbodens - Vergleich von Drucksondierungen in der Kalibrationskammer mit undranierten zyklischen Triaxialversuchen, „Karlsruhe, 2017.

[8] O. V. Salas, «Ensayos triaxiales para suelos,» San José, 2011.

[9] C. V. Moreno, «Análisis de licuación de arenas mediante el empleo de métodos de campo, análisis de respuesta de sitio y modelos numéricos de generación de presión de poro,» México DF, 2015.

[10] L. Youd, M. Idriss, R. Andrus, I. Arango, G. Castro, J. Christian, R. Dobry, L. Finn, L. Harder, M. E. Hynes, K. Ishihara, J. Koester, S. Liao, W. Marcuson, G. Martin y Mitchell, «LIQUEFACTION RESISTANCE OF SOILS: SUMMARY REPORT FROM THE 1996 NCEER AND 1998 NCEER/NSF WORKSHOPS ON EVALUATION OF LIQUEFACTION RESISTANCE OF SOILS,» 2001.

[11] M. C. Scholz, «Entwicklung von Korrelationen zur Ermittlung des Verflussigungswiderstandes von Sand in Tagebau-Restseeboschungen auf der Basis von Drucksondierungen, » Karlsruhe, 2017.

[12] S. T. у T. W., «Teparaksa. Evaluation of liquefaction potential of soils using cone penetration tests, 1988.

[13] D. W. a. W. F. P.K. Robertson, «Seismic cone penetration test for cone penetration test for evaluating liquefaction potential under cyclic loading, » Canadian Geotechnical Journal, pp. 686-685, 1992.

[14] B. Seed y H. Alba, «Use of SPT and CPT tests for evaluating the liquefaction resistance of sands, Geotechnical Special Publication 6, pp. 281-302, 1986. 